\title{
Therapeutic targets during mitochondrial lipid metabolism
}

\author{
William Wang • Liyang Li $\mathbb{D} \cdot$ Xiangdong Wang
}

Received: 24 May 2020 / Accepted: 11 June 2020 / Published online: 17 June 2020

(C) Springer Nature B.V. 2020

Mitochondrial lipid metabolism plays a central role in the regulation of cell function via bi-directional signaling, e.g., mitochondrial dysfunction altering intra- and inter-cellular lipid metabolism, while cytosolic disorder of lipid production compromises mitochondrial lipid metabolism. Mitochondria-dominated dysfunction, i.e., oxidative stress, lipid toxicity, telomere shortening, metabolic disturbance, and DNA damage, contributes to cell autophagy, mitochondria-selective autophagy (mitophagy), ferroptosis, pyroptosis, apoptosis, and necroptosis in the development of cell toxicity, chronic diseases, and metabolism disorders (Fang et al. 2019; Li et al. 2020; Khatami 2018; Wu et al. 2018). Jain et al. (2020) found that mitochondrial and lipid metabolism plays a critical role in the maintenance of cell fitness in oxidation stress, during which the fatty acid metabolism in mitochondrial oxidative phosphorylation or etherlipid metabolism in peroxisome gene sets were selected by genetic screening as the core signal pathways in low or high oxygen stresses. Of mitochondrial multi-functions, mitochondrial fusion (mtFusion) and fission (mtFission) were also named "dynamics" and are considered as the quality control pathways in the maintenance of cell death and proliferation, especially mitophagy. Mitochondrial morphology and biofunction are highly

W. Wang $\cdot$ L. Li $\cdot$ X. Wang $(\bowtie)$

Zhongshan Hospital Institute for Clinical Science, Shanghai Institute of Clinical Bioinformatics, Shanghai Engineering Research for AI Technology for Cardiopulmonary Diseases, Fudan University, Shanghai, China e-mail: xdwang@clintranslmed.org dependent upon the regulation and control of mtFusion and mtFission. Large number of specific mitoproteases, proteins, as well as inner- and outer-mitochondrial membrane GTPases control and regulate the dynamic process of mitochondrial fusion and fission, including mitochondrial Lon, caseinolytic protease $\mathrm{P}$, inner membrane proteins and peptidases, rhomboid protease, PTEN-induced putative protein kinase 1, dynaminrelated protein 1 (Drp1), fission-1, mitochondrial fission factor, and mitofusin (Mfn). In addition, the intra- and extra-mitochondrial lipid metabolism and lipid elements play an important role in the process of mitochondrial dynamics. Phosphatidic acid and cardiolipin can interact with those control factors to maintain the process of $\mathrm{mtFusion} / \mathrm{mtFission}$. Thus, it is crucial to explore the interaction between $\mathrm{mtFusion} / \mathrm{mtFission}$ with lipid metabolisms, developing further understanding in the influence of lipid elements in the process of mitochondrial dynamics, and how to define the molecular control of mitochondrial membrane movements.

Interaction and regulation between mtFusion/ mtFission and lipids maintain mitochondrial function and stability, cytoplasmic homeostasis, and cell structure and function. The mitochondrial dynamics changes the patterns of lipid metabolism. Sênos Demarco et al. (2019) demonstrated that mtFusion could be a decisive factor in the differentiation of germline stem cells under the regulation of Mfns or optic atrophy 1 (Opa1) through a feedback circle of mitochondrial function and lipid drops. During mtFusion, the outer mitochondrial membrane fusion is mediated by Mfn1 and $\mathrm{Mfn} 2$ and then the inner membrane fusion occurs in 
regulation of Opa1. This process depends upon the distribution of membrane lipid composition, intra- or extra-mitochondrial lipid flows, or other factors modulated by post-translational modifications and mitochondrial DNA methylation (Wang et al. 2020). The homology regions-2 and/or GTPase domains of Mfns on the outer membrane of two opposing mitochondria interacted by Opa1-dependent GTPase binding or/and GTP hydrolysis, docking and exploring membrane contact sites, and dimerizing and inducing Mfns dimerization and conformational changes. The out membrane is fused and regulated by phospholipids, then the inner membrane starts to be fused by the interaction between Opa1 and cardiolipin. The process of $\mathrm{mtFusion}$ can increase the accumulation of lipid droplets and the utilization of lipids by constitutive activation of the target of rapamycinpathway and lipogenesis. Importantly, mtFusionincreased cytoplasmic lipids may alter the function of metabolic regulators, histones, and mitochondria, downregulating fatty acid oxidation and converting excessive fatty acids into triacylglycerides in lipid droplets. The activation of palmitoyltransferase 1 and fatty acid oxidation can promote the importing of fatty acids into mitochondria, dependent upon the presence of Mfn or Opa1. On the other hand, those secondary changes of lipid droplets can dynamically interact with other organelles like endoplasmic reticulum, phagosomes, peroxisomes, and mitochondria, regulating the process of mtFusion. Their transient and rapid morphological adaptations are crucial for many cellular processes such as the cell cycle, immunity, apoptosis, and mitochondrial quality control. Mutations in the core machinery components and defects in the mitochondrial dynamics have been associated with numerous human diseases. These dynamic transitions are mainly ensured by large GTPases belonging to the Dynamin family. Mitochondrial fission is a multi-step process allowing the division of one mitochondrion into two daughter mitochondria. It is regulated by the recruitment of the GTPase Drp1 by adaptors at actin- and endoplasmic reticulum-mediated mitochondrial constriction sites. Drp1 oligomerization followed by mitochondrial constriction can recruit Dynamin 2 to stop membrane scission.

Mitochondria-driven lipid is an important regulatory source that plays an important role in the intercommunication between intracellular organelles and is one of the lipid sources for lipid droplets. Lipid droplets connect dynamic organelles rich of triacylglycerols between membrane leaflets, surrounded with a lipid monolayer and proteins. Lipid droplets contribute to intra-cellular and inter-organellar energy metabolism, membrane integration, and microenvironmental homeostasis. In addition to its roles in mtFusion and mtFission, mitoguardin-2 as the outer mitochondrial membrane protein is anchored to mitochondria by two amphipathic transmembrane segments at the $\mathrm{N}$ terminus to regulate $\mathrm{mtFusion} / \mathrm{mtFission}$ function-associated metabolism and to bridge between mitochondria and lipid droplets, between mitochondria and endogenous reticulum, and between endogenous reticulum and lipid droplets in adipocytes for interorganellar communication (Freyre et al. 2019). Intercommunication among organelles is highly dependent upon the binding capacity of the outer mitochondrial membrane proteins with other membrane proteins like vesicle-associated membrane protein-associated proteins and translocase of outer membrane 40. Mitochondriadriven triglyceride synthesis and storage are involved in the process from de novo lipogenesis in mitochondria to triglyceride production in the endogenous reticulum through the activation of mitochondria membrane proteins. Those mitochondrial proteins are responsible for the regulation of cytosolic lipid and protein homeostasis, mitophagy, and cell integrity. It remains unclear whether mitochondria-driven lipids act as the signaling messages to guide, promote, or initiate the intercommunication among organelles, as the catalyzer to activate membrane proteins or reshape organelles, or as the buffer to neutralize phosphorylation, S-nitrosylation, SUMOylation, OGLcNAcylation, acetylation, or ubiquitination. The interaction between cytolytic lipids and mitochondrial dynamics plays a decisive role in lipid metabolism, mitochondrial function, and cell survival, while the specificity and pattern of such effects should be furthermore clarified in diseases. For example, the microenvironmental homeostasis of intra- and inter-cancer cells changes and varies with cancer types, stages, durations, and responses to drugs (Agrawal 2019; Kadel et al. 2019), The shape and function of homology regions-1and 2 domains can differ between healthy and cancer cells, resulting in dysfunction of lipid metabolism secondary to altered regulations of mtFusion and mtFission and an increase of chemical and biophysical lipids variation in membrane composition of endoplasmic reticulum and mitochondria.

Dysfunction of mtFusion/mtFission, energy transport, and mitophagy is responsible for mitochondriadependent or non-dependent diseases. The disorders and imbalance of mtFusion and mtFission can be one of the molecular mechanisms responsible for the 
initiation and progression of diseases. The disruption and impairment of mitochondrial dynamics are associated with clinical phenotypes of patients with neurodegenerative diseases, e.g., Alzheimer's, Huntington's, and Parkinson's diseases. For example, of neurodegenerative diseases, Charcot-Marie-Tooth type 2A is mainly caused by the primary deficiency of mitochondrial out membrane fusion due to Mfn2, dominant optic atrophy by inner membrane fusion due to Opa1, neuro-degeneration and early death by mtFission due to Drp1, and Parkinson's disease by mitophagy due to PARK2 and PINK1 (Meyer et al. 2017). The balance of mtFusion and mtFission also plays a role in the regulation of cell sensitivity to intercellular signals like inflammatory mediators, to environmental chemicals like toxicants, and to therapeutic agents like drugs. Genetic alterations of mitochondria-associated genes can lead to inherited/ congenital dysfunction of mtFusion/mtFission or change cell sensitivity and susceptibility to challenges. Exogenous toxicants and drugs can induce the mutation and epigenetic modifications of mitochondria-associated genes, causing the secondary dysfunction of mtFusion/ mtFission. We should investigate the potential effects between the secondary and primary mtFusion/ mtFission compromise, since impaired mitochondrial dynamics could alter the capacity of stem cell selfrenew or differentiation (Sênos Demarco et al. 2019).

Impaired mitochondrial dynamics increased cell susceptibility to challenges through altered lipid metabolism. Chung et al. (2019) found that animals with the single-gene deletion of Mfn1 or Mfn2 in lung alveolar type 2 epithelial cells had normal lungs, while rapidly developed bleomycin-induced lung fibrosis. Deletion of both Mfn1 and Mfn2 caused animal spontaneous lung fibrosis and death at an early stage of the development, which failed to occur in animals with the single deletion. This indicates that those animals became more sensitive to the challenge, and one Mfns have enough function to maintain mitochondrial function by a compensative mechanism. Mfns play decisive role in the process of $\mathrm{mtFusion/mtFission,} \mathrm{lipid} \mathrm{metabolism,} \mathrm{and} \mathrm{cell} \mathrm{re-}$ sponse to mitochondrial injury, while their deletion impaired regulation of lipid metabolism, e.g., basal surfactant phospholipid and cholesterol metabolism, leading to the development of spontaneous lung fibrosis. The peroxisomal factor Pex16 has the capacity of maintaining cell tolerance to cold, energy expenditure, mtFission, mtDNA copy number, and mitochondrial function through peroxisomal lipid metabolism and biogenesis like fatty acid oxidation and plasmalogen synthesis regulated by the plasmalogen synthetic enzyme glyceronephosphate O-acyltransferase (Park et al. 2019). It seems that plasmalogens present in mitochondria can regulate and control cell tolerance through Pex16 activation and mitochondrial dynamics, and thermogenesis can be regulated by the interaction between peroxisomes and mitochondria.

The interaction and regulation between mtFusion/ mtFission play a key role in lipid metabolism control and the maintenance of mitochondrial stability and cytoplasmic homeostasis. It also contributes to both mitochondrial and cellular morphology and function. Mitochondria dynamics-associated lipid metabolism can regulate the intercommunication between organelles and is required for lipid droplets. The balance between $\mathrm{mtFusion} / \mathrm{mtFission}$ is required in the regulatory effects of lipid droplets among organelles. Dysfunction of mtFusion/mtFission, energy transport, and mitophagy is responsible for the mitochondria-dependent or nondependent diseases. Impaired mitochondrial dynamics increased cell susceptibility and tolerance to challenges through altered lipid metabolism. Thus, a deep understanding of the interaction between mitochondrial dynamics and lipid metabolisms will provide insight into the molecular mechanisms of mitochondria-associated diseases and into the development of biomarkers and therapeutic targets.

\section{References}

Agrawal B. New therapeutic targets for cancer: the interplay between immune and metabolic checkpoints and gut microbiota. Clin Transl Med. 2019;8(1):23. https://doi.org/10.1186 /s40169-019-0241-x.

Chung KP, Hsu CL, Fan LC, Huang Z, Bhatia D, Chen YJ, et al. Mitofusins regulate lipid metabolism to mediate the development of lung fibrosis. Nat Commun. 2019;10(1):3390. https://doi.org/10.1038/s41467-019-11327-1.

Fang T, Wang M, Xiao H, Wei X. Mitochondrial dysfunction and chronic lung disease. Cell Biol Toxicol. 2019;35(6):493502. https://doi.org/10.1007/s10565-019-09473-9.

Freyre CAC, Rauher PC, Ejsing CS, Klemm RW. MIGA2 links mitochondria, the ER, and lipid droplets and promotes de novo lipogenesis in adipocytes. Mol Cell. 2019;76(5):811825.e14. https://doi.org/10.1016/j.molcel.2019.09.011.

Jain IH, Calvo SE, Markhard AL, Skinner OS, To TL, Ast T, et al. Genetic screen for cell fitness in high or low oxygen highlights mitochondrial and lipid metabolism. Cell. 2020;181(3):716-727.e11. https://doi.org/10.1016/j. cell.2020.03.029. 
Kadel D, Zhang Y, Sun HR, Zhao Y, Dong QZ, Qin LX. Current perspectives of cancer-associated fibroblast in therapeutic resistance: potential mechanism and future strategy. Cell Biol Toxicol. 2019;35(5):407-21. https://doi.org/10.1007 /s10565-019-09461-z.

Khatami M. Cancer; an induced disease of twentieth century! Induction of tolerance, increased entropy and 'Dark Energy': loss of biorhythms (Anabolism v. Catabolism). Clin Transl Med. 2018;7(1):20. https://doi.org/10.1186 /s40169-018-0193-6.

Li L, Bi Z, Hu Y, Sun L, Song Y, Chen S, et al. Silver nanoparticles and silver ions cause inflammatory response through induction of cell necrosis and the release of mitochondria in vivo and in vitro. Cell Biol Toxicol. 2020. https://doi. org/10.1007/s10565-020-09526-4.

Meyer JN, Leuthner TC, Luz AL. Mitochondrial fusion, fission, and mitochondrial toxicity. Toxicology. 2017;391:42-53. https://doi.org/10.1016/j.tox.2017.07.019.

Park H, He A, Tan M, Johnson JM, Dean JM, Pietka TA, et al. Peroxisome-derived lipids regulate adipose thermogenesis by mediating cold-induced mitochondrial fission. J Clin Invest. 2019;129(2):694-711. https://doi.org/10.1172/JCI120606.

Sênos Demarco R, Uyemura BS, D'Alterio C, Jones DL. Mitochondrial fusion regulates lipid homeostasis and stem cell maintenance in the drosophila testis. Nat Cell Biol. 2019;21(6):710-20. https://doi.org/10.1038/s41556-0190332-3.

Wang W, Liu X, Wang X. How to breakthrough mitochondrial DNA methylation-associated networks. Cell Biol Toxicol. 2020.

Wu D, Wang X, Sun H. The role of mitochondria in cellular toxicity as a potential drug target. Cell Biol Toxicol. 2018;34(2):87-91. https://doi.org/10.1007/s10565-0189425-1.

Publisher's note Springer Nature remains neutral with regard to jurisdictional claims in published maps and institutional affiliations. 